1. Charny CK, Stanziale SF, Khilnani NM, Helfet DL, Ezchempati SR, Barie PS. Unstable pelvic fracture and massive retroperitoneal hematoma from transection of the superior gluteal artery. J Trauma 2000; 48: 359.

2. Chen AL, Wolinsky PR, Tejwani NC. Hypogastric artery disruption associated with acetabular fracture. A report of two cases. J Bone Joint Surg Am 2003; 85-A: 333-8.

3. Velmahos GC, Toutouzas KG, Vassiliu P, Sarkisyan G, Chan LS, Hanks SH, et al. A prospective study on the safety and efficacy of angiographic embolization for pelvic and visceral injuries. J Trauma 2002; 53: 303-8

4. Kimbrell BJ, Velmahos CG, Chan LS, Demetriades D. Angiographic embolization for pelvic fractures in older patients. Arch Surg 2004; 139: 728-32

5. Velmhaos GC, Chahwan S, Hanks SE, Murray JA, Berne TV, Asensio $\mathrm{J}$, Demetriades D. Angiographic embolization of bilateral internal iliac arteries to control life-threatening hemorrhage after blunt trauma to the pelvis. Am Surg 2000; 66: 858-62.

6. Wong YC, Wang LJ, Ng CJ, Tseng IC, See LC. Mortality after successful transcatheter arterial embolization in patients with unstable pelvic fractures: rate of blood transfusion as a predictive factor. Trauma 2000; 49: 71-5.

7. Holting T, Buhr HJ, Richter GM, Roeren T, Friedl W. Diagnosis and treatment of retroperitoneal hematoma in multiple trauma patients. Arch Orthop Trauma Surg 1992; 111: 323-6.

8. Pieri S, Agresti M, Morucci M, De Medici M, Galluzzo M, Oransky M. Percutaneous management of hemorrhages in pelvic fractures. Radiol Med 2004; 107: 241-51.

9. Cook RE, Keating JF, Gillespie I. The role of angiography in the management of haemorrhage from major fractures of the pelvis. J Bone Joint Surg Br 2002; 84: 178-82.

10. Sriussadaporn S, Sirichindakul B, Pak-Art R., Tharavej C. Pelvic fractures: experience in management of 170 cases at a university hospital in Thailand. J Med Assoc Thai 2002; 85: 200-6.

\section{Diagnóstico simultáneo de adenocarcinoma y GIST gástricos}

\section{Sr. Director:}

El 60\% de los tumores del estroma gastrointestinal (gastrointestinal stromal tumors -GIST-) se originan en el estómago, si bien sólo suponen el $2 \%$ de las neoplasias gástricas. Recientemente se han publicado casos de pacientes en que el GIST ha aparecido de manera sincrónica o sucesiva a otros tumores diges- tivos con diferente grado de agresividad (1-3). A los casos publicados hasta la fecha concretamente de diagnóstico simultáneo de afectación gástrica por GIST y adenocarcinoma (4-8), añadimos nuestra experiencia con una paciente.

Mujer de 77 años, sin antecedentes de interés, que ingresó para estudio de anemia crónica (hemoglobina 5,2 g/dl, VCM 62 fl). La gastroscopia mostró una lesión ulcerada de bordes irregulares en la cara anterior del estómago. La biopsia resultó compatible con adenocarcinoma. No se encontró evidencia de afectación a distancia. En la laparotomía se apreció una neoplasia en el antro y una pequeña tumoración mesenquimal subserosa de un centímetro de diámetro. Se practicó una gastrectomía subtotal. El informe histológico fue de adenocarcinoma de tipo intestinal bien diferenciado intramucoso ulcerado. La lesión mesenquimal resultó ser un tumor del estroma gastrointestinal (GIST) con marcada positividad para CD-117 y CD-34 y con menos de cinco mitosis por cada 50 campos de gran aumento (CGA). Se aislaron cuatro ganglios, todo ellos libres de infiltración neoplásica. No se encontraron signos de infección por Helicobacter pylori. A los seis meses de la intervención la paciente continúa viva y sin evidencia de recidiva.

El interés por el estudio de los GIST ha crecido desde que se demostró la eficacia del imatinib en el tratamiento de estos tumores. En los últimos años se han publicado nueve casos, incluido el que presentamos, todos ellos de pacientes de edad superior a 70 años, en que coincidió el diagnóstico de GIST (confirmado mediante al menos una técnica inmunohistoquímicaix: CD 34 y/o CD117), con el de adenocarcinoma gástrico (4-8) (Tabla I). En uno de ellos se apreciaba incluso una imbricación ("colisión") de las dos histologías tumorales (6). Hay autores que han especulado con un origen biológico común de los diferentes tumores gástricos: adenocarcinoma, linfoma tipo MALT e incluso GIST $(4,6)$. Sin embargo, esta posibilidad, por ahora, no ha pasado de ser una mera hipótesis. De hecho, es probable que la presencia simultánea de GIST y adenocarcinoma gástricos sea tan solo un mero hallazgo incidental. Esta posibilidad viene respaldada por el hecho de que la mayoría de los GIST que se han encontrado en estos casos han sido tumores de buen pronóstico (9), es decir, de pequeño tamaño y con bajo número de mitosis, algo que se traslada en un comportamiento relativamente indolente. De hecho, en clasificaciones antiguas estos tumores del estroma gástrico eran catalogados como "benignos" por su crecimiento lento y su escasa tendencia a diseminarse a distancia $(4,10)$. En todo caso, es probable que el empleo de técnicas de inmunohistoquímica relativamente específicas facilite el diagnóstico de más casos de presencia simultánea de GIST y adenocarcinoma gástricos. Esto puede ayudar a conocer si realmente existe alguna asociación o si la coincidencia de dos tumores se trata tan sólo de un hallazgo incidental.

TABLA I

CASOS DE DIAGNÓSTICO SIMULTÁNEO DE ADENOCARCINOMA Y GIST GÁSTRICOS

\begin{tabular}{|c|c|c|c|c|c|c|c|c|}
\hline \multirow[t]{2}{*}{ Ref. } & \multicolumn{2}{|c|}{ Paciente } & \multicolumn{2}{|c|}{ Adenocarcinoma } & \multicolumn{2}{|c|}{ GIST } & \multirow[t]{2}{*}{ H. pylori } & \multirow[t]{2}{*}{ Otros tumores } \\
\hline & Sexo & Edad & Grado & Estadio & $\begin{array}{c}\text { Tamaño } \\
\text { (cm) }\end{array}$ & $\begin{array}{l}\text { Mitosis } \\
(50 \text { CGA) }\end{array}$ & & \\
\hline 4 & Mujer & 79 & & T1N0 & 6,0 & $>5$ & Sí & \\
\hline 4 & Varón & 79 & & T2NO & 0,6 & $<5$ & No & \\
\hline 5 & Varón & 72 & & $\mathrm{~T} 2 \mathrm{NO}$ & 2,5 & $<1$ & Sí & Adenocarcinoma de vesícula biliar \\
\hline 6 & Varón & 70 & & T3N1 & & 0 & No & \\
\hline 8 & Varón & 77 & G2 & $\mathrm{T} 2 \mathrm{~N} 1$ & 0,6 & 0 & No & \\
\hline Actual & Mujer & 77 & G1 & T1N0 & 1,0 & $<5$ & No & \\
\hline
\end{tabular}


A. S. Rubiales, Y. Ovelar ${ }^{1}$, J. Beltrán de Heredia ${ }^{2}$, M. L. del Valle

Servicios de Oncología, ${ }^{I}$ Anatomía Patológica $y{ }^{2}$ Cirugía General. Hospital Clínico Universitario. Valladolid

1. Nakaya I, Iwata Y, Abe T, Yokohama H, Oda Y, Nomura G. Malignant gastrointestinal stromal tumor originating in the lesser omentum, complicated by rapidly progressive glomerulonephritis and gastric carcinoma. Intern Med 2004; 43: 102-5.

2. Kover E, Faluhelyi Z, Bogner B, Kalmar K, Horvath G, Tornoczky T. Dual tumours in the gastrointestinal tract: synchronous and metachronous stromal (GIST) and epithelial/neuroendocrine neoplasms. Magy Onkol 2004; 48: 315-21.

3. Sailors JL, French SW. The unique simultaneous occurrence of granular cell tumor, gastrointestinal stromal tumor, and gastric adenocarcinoma. Arch Pathol Lab Med 2005; 129: 121-3.

4. Maiorana A, Fante R, Maria Cesinaro A, Adriana Fano R. Synchronous occurrence of epithelial and stromal tumors in the stomach: a report of 6 cases. Arch Pathol Lab Med 2000; 124: 682-6.

5. Chen JH, Chen CC, Tzeng LM, Tsay SH, Chiang JH, Lu CC, et al. Resection of triple synchronous tumors - gastric adenocarcinoma, gallbladder adenocarcinoma and stromal tumor of the stomach. Zhonghua Yi Xue Za Zhi (Taipei) 2001; 64: 655-60.

6. Liu SW, Chen GH, Hsieh PP. Collision tumor of the stomach: a case report of mixed gastrointestinal stromal tumor and adenocarcinoma. J Clin Gastroenterol 2002; 35: 332-4.

7. Kaffes A, Hughes L, Hollinshead J, Katelaris P. Synchronous primary adenocarcinoma, mucosa-associated lymphoid tissue lymphoma and a gastrointestinal stromal tumor in a Helicobacter pylori-infected stomach. J Gastroenterol Hepatol 2002; 17: 1033-6.

8. Bircan S, Candir O, Aydin S, Baspinar S, Bulbul M, Kapucuoglu N, et al. Synchronous primary adenocarcinoma and gastrointestinal stromal tumor in the stomach. A report of two cases. Turk J Gastroenterol 2004; 15: 187-91.

9. Corless CL, Fletcher JA, Heinrich MC. Biology of gastrointestinal stromal tumors. J Clin Oncol 2004; 22: 3813-25.

10. Ma CK, Amin MB, Kintanar E, Linden MD, Zarbo RJ. Immunohistologic characterization of gastrointestinal stromal tumors: a study of 82 cases compared with 11 cases of leiomyomas. Mod Pathol 1993; 6: 13944.

\section{Sobredosis de cianamida cálcica (carbimida): descripción de un caso y revisión bibliográfica}

\section{Sr. Director:}

La carbimida, o cianamida cálcica (fórmula molecular CaCN2), es utilizada en el tratamiento de la deshabituación de los pacientes alcohólicos crónicos ya que ayuda a mantener la abstinencia de provocando en los pacientes aversión hacia el alcohol, de forma similar al disulfiram $(1,2)$. Actúa inhibiendo a nivel hepático la aldehído-deshidrogenasa (ALDH) encargada de la oxidación del principal metabolito del etanol, el acetaldehído. El bloqueo de la ALDH en presencia de etanol en sangre provoca un incremento de acetaldehído, que se traduce clínicamente en lo que se conoce como reacción aldehídica o efecto antabús, caracterizada por una vasodilatación brusca con enrojecimiento facial intenso, palpitaciones, sudoración, visión borrosa, náuseas, taquicardia, dificultad respiratoria y debilidad generalizada. Las reac- ciones más graves pueden producir hipotensión, depresión respiratoria y colapso cardiocirculatorio (1).

En ausencia de alcohol la carbimida es bien tolerada. Con el uso crónico se han comunicado ocasionales efectos secundarios del tipo de mareos, dermatitis de contacto, hepatitis, granulopenia, etc. (1,3-5), pero la toxicidad aguda por sobredosificación de carbimida es poco conocida ya que existen pocos casos recogidos en la literatura (5) (descriptores: cyanamide/poisoning Medline: 1966-2004). Presentamos el caso de una paciente que ingirió carbimida de forma masiva con finalidad autolítica.

Paciente mujer de 38 años con antecedentes de esquizofrenia y bulimia. Fumadora de 90 cigarrillos/día. Historia de abuso alcohólico y tratamiento deshabituador, hasta hacía 2 años. Ictus isquémico en julio de 2004, con recuperación sin secuelas. Seguía tratamiento con olanzapina oral y trifusal. Se encontraba en fase estable de su proceso psiquiátrico y su régimen de vida no presentaba limitaciones importantes. Historia de alergias, cardiopatía, broncopatía o hepatopatía, negativa.

Llevada a Urgencias por presentar de forma brusca nauseas y disminución del nivel de conciencia, sin responder a órdenes verbales y con escasa respuesta al dolor. En la exploración física, inconsciente con tensión arterial 104/52 mm de $\mathrm{Hg}$, frecuencia cardiaca a 105 latidos por minuto, frecuencia respiratoria 34 respiraciones por minuto y temperatura $37^{\circ} \mathrm{C}$. Reaccionaba perezosamente al dolor, sin apertura ocular. Emitía sonidos ininteligibles. Pupilas isocóricas y normorreactivas. Reflejos cutáneos plantares, flexores de forma bilateral. Taquipnéica. No ingurgitación yugular, no adenopatías. Auscultación cardiaca rítmica, sin soplos. Auscultación pulmonar, roncus en bases. Abdomen y extremidades sin anormalidades. Ausencia de fetor alcohólico o cetonémico. Se realizó lavado gástrico sin observarse restos de restos de fármacos. Hemograma: hematocrito 0,36 1/1, hemoglo-

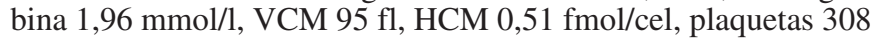
x $10^{9} / 1$, Leucocitos 10,1 x 10\% $/$. Bioquímica sérica: glucosa 10,2 $\mathrm{mmol} / \mathrm{l}$, urea $6 \mathrm{mmol} / \mathrm{l}$, creatinina $106 \mathrm{mmol} / \mathrm{L}$, sodio 132 $\mathrm{mmol} / \mathrm{L}$, potasio $4,9 \mathrm{mmol} / \mathrm{L}$, calcio $2,2 \mathrm{mmol} / \mathrm{L}, \mathrm{CK} 96 \mathrm{U} / \mathrm{L}$, AST 37 U/L. Orina y sedimento normales. $\mathrm{pH}$ urinario 5,5. Sodio en orina $184 \mathrm{mmol} / \mathrm{l}$, potasio $21 \mathrm{mmol} / \mathrm{l}$, cloro $194 \mathrm{mmol} / \mathrm{l}$. Estudio de coagulación normal. Triaje de drogas en orina negativo para anfetaminas, metanfetaminas, barbitúricos, benzodiacepinas, cannabinoides, cocaína, opiáceos, fenciclidina y antidepresivos tricíclicos. Análisis de líquido cefalorraquídeo normal. Radiografía de tórax y ecografía abdominal normales. TC craneal con lesión hipodensa parietal izquierda presumiblemente isquémica sin otros hallazgos. Las determinaciones gasométricas se pueden observar en la tabla I. Recibió tratamiento antibiótico por sospecha de infección respiratoria y tratamiento broncodilatador y oxigenoterapia. Para el trastorno metabólico recibió tratamiento con 1,5 gramos/día de bicarbonato por vía oral a partir del tercer día de ingreso. Su estado de conciencia mejoró progresivamente hasta la normalidad, encontrándose en su estado habitual al séptimo día del ingreso, momento en el que reconoció la ingesta de 4 envases de Colme® (3.600 mg). En el momento del alta hospita-

\section{TABLA I}

EVOLUCIÓN DE LA ACIDOSIS. DETERMINACIONES GASOMÉTRICAS

\begin{tabular}{lccccc}
\hline & $p \mathrm{H}$ & $\begin{array}{c}\mathrm{PO}_{2} \\
(\mathrm{~mm} \mathrm{Hg})\end{array}$ & $\begin{array}{c}\mathrm{PCO}_{2} \\
(\mathrm{~mm} \mathrm{Hg})\end{array}$ & $\begin{array}{c}\text { Bicarbonato } \\
(\mathrm{mmol} / \mathrm{L})\end{array}$ & $\begin{array}{c}\text { Exceso de } \\
\text { bases }\end{array}$ \\
\hline Urgencias* $^{*}$ & 7,44 & 69 & 29 & 20 & $-2,6$ \\
24 horas* $^{*}$ & 7,33 & 90 & 33 & 18 & $-7,8$ \\
72 horas* & 7,32 & 111 & 27 & 13,5 & $-10,8$ \\
$5^{\circ}$ dia $^{*}$ & 7,38 & 70 & 34 & 21 & -4 \\
$10^{\circ}$ dia $^{*}$ & 7,36 & 23 & 49 & 27,7 & +2 \\
\hline
\end{tabular}

\footnotetext{
*Gasometría arterial. *Gasometría venosa.
} 